Original research

\title{
Observational placement: a useful learning tool to consolidate wound management learning amongst podiatry students
}

\section{Henshaw FR and Qasem S}

Keywords wounds, management, student, placement, observe

For referencing Henshaw FR \& Qasem S. Observational placement: a useful learning tool to consolidate wound management learning amongst podiatry students. Wound Practice and Research 2020; 28(4):163-167.

DOI https://doi.org/10.33235/wpr.28.4.163-167

\section{Abstract}

Introduction Feedback from placement supervisors has shown that podiatry students are often overwhelmed and lack confidence when placed in complex clinical environments such as high risk foot clinics. Existing literature suggests that student anxiety on placement can reduce the value of learning attained. It is therefore logical that observation-only placement, in the earlier phases of learning, may assist with bridging the gap between theoretical learning and practical application. Currently, limited exists evidence to support this supposition.

Methods and materials 3rd year podiatry students attended a half-day observation at a high risk foot clinic and subsequently completed an online survey following the placement to evaluate their experience. This evaluation

\section{Frances R Henshaw*}

B Sc (hons) PhD

School of Science and Health, Western Sydney

University, Penrith, NSW, Australia

Translational Health Research Institute, Western

Sydney University, Penrith, NSW, Australia

Email f.henshaw@westernsydney.edu.au

\section{Saja Qasem}

B.Hlth Sc M.Pod Med

School of Science and Health, Western Sydney

University, Penrith, NSW, Australia

Podiatrist, High Risk Foot Service, Royal North

Shore Hospital, Sydney, NSW, Australia

* Corresponding author was consistent with Levels I/II of Kirkpatrick's four levels of analysis, examining the degree to which participants found the placement relevant to their learning (Level I), and acquired the intended knowledge, and confidence (Level II).

Results $88 \%$ of students agreed that they felt more confident in their clinical abilities, and $94 \%$ of students agreed that their understanding of podiatrists' scope of practice was improved following the observational placement.

Conclusions Inclusion of observational placements within the pre-clinical phase of allied health degrees should be considered as they improve student confidence and can be helpful in consolidation of learning.

\section{Introduction}

Clinical placement is a cornerstone of vocational learning in health professions ${ }^{1}$, yet evidence suggests that clinical environments can be stressful to students ${ }^{2-4}$. Self-confidence has been identified to be an essential aspect required for clinical learning and performance ${ }^{5}$. It is known that the initial stages of clinical placements are anxiety provoking ${ }^{6}$. Sharif and Masoumi ${ }^{6}$ attributed this anxiety to a lack of familiarity with the clinical environment, compounded by a lack of clinical skill. Further research has confirmed that confidence empowers students to become more competent clinicians ${ }^{7}$. Conversely, when students lack confidence and self-belief, their cognition may be impaired and, consequently, patient safety may be compromised ${ }^{8}$.

In Australia, students enrolled in podiatry programs are required to complete about 1000 hours of clinical placement in order to meet national accreditation standards ${ }^{9}$. This includes attaining competency in the assessment and management of complex wound-related foot problems such as diabetic foot ulcers, venous leg ulcers and osteomyelitis. Traditionally, 
students acquire knowledge through a combination of didactic learning and high-fidelity simulation. These methods are effective for acquiring clinical skill; however, they do not prepare students for the true stressors of a complex clinical environment.

Feedback from several providers of podiatry clinical placements within public hospital facilities (personal communication) has been that students frequently feel overwhelmed when faced with complex clinical cases, such as diabetic foot ulcers, which can be both limband life-threatening. Clinical placements are seen as a prime opportunity to consolidate theoretical learning through practical application; however, learning can be hindered due to student anxiety levels and a lack of confidence ${ }^{8}$. It is therefore reasonable that the incorporation of observationonly placement, in the early phases of learning, might help students to consolidate their theoretical learning by observing episodes of complex care being delivered by their more experienced peers. However, evidence to support observational-only placements as a means to improve student clinical learning is currently lacking. Therefore, the aim of this study is to evaluate observation-only placements as a means to improve podiatry student knowledge and confidence within complex clinical environments via a postobservational placement survey.

\section{Methods and materials}

A half day observational placement was incorporated into the 'High Risk Foot', a 3rd year unit undertaken by podiatry students at Western Sydney University. Prior to attending this placement, all participants completed a number of lectures and tutorials covering theory on the assessment and management of complex lower limb problems; this comprised approximately 20 hours of face-to-face teaching. The teaching articulated with four learning objectives that are prescribed in the university-approved unit outline for the 'High Risk Foot' subject:

- Execute a thorough examination of the lower extremity vasculature, diagnose vascular status, recognise limitations of these examinations during interpretation of results and instigate an appropriate management plan with an emphasis on the role of the podiatrist in the interdisciplinary health care team.

- Describe the physiological mechanisms of wound healing, in the presence or absence of normal physiological response and thorough examination and investigations and ascertain the underlying causes of the wound, and generate and implement appropriate strategies to return the tissues to their most functional levels by addressing both the wound and the underlying causes.

- Discuss the physiological basis of the clinical manifestations of diabetes mellitus as it affects multiple organ and functional systems by performing appropriate examinations (vascular, neurological, musculoskeletal, dermatological and psychosocial).

- Diagnose, evaluate and treat the foot conditions presented by the person with diabetes mellitus, in particular dermal ulcers and explain the role of physical therapies, particularly footwear and orthotic therapy in the ongoing patient management, and health education and promotion strategies.

Students, in groups of four, were assigned to attend a halfday placement at a high risk foot clinic, where complex limbthreatening problems such as wounds and osteomyelitis are managed. The clinic is a podiatrist-led, multi-disciplinary outpatient facility within a tertiary public hospital in the Sydney metropolitan area. Following a 30-minute induction provided by a designated placement coordinator (a podiatrist), students were assigned to shadow a clinician for the remainder of the session ( 3 hours) at a ratio of between one to three students per clinician.

Immediately following the placement, students were asked to complete an online custom-designed survey via an online platform called Respondster (Respondster.com) to measure overall placement satisfaction as well as self-rated confidence and knowledge levels. Students were asked to evaluate their experiences on a five-point scale. Text boxes were provided to enable participants to provide more detailed feedback.

Kirkpatrick's model of teaching evaluation was used to appraise the training outcomes of the placement. The model is one of the most well-known and widely used evaluation models for training and development programs ${ }^{10}$. It provides four levels of training outcomes - reaction, learning, behaviour and results, to create an effective and objective framework for the evaluation of educational training programs ${ }^{11}$. The model enables the user to evaluate whether a program meets the intended need ${ }^{12}$. Kirkpatrick's model is widely used in assessment of clinical placements ${ }^{13-15}$ and was therefore selected as an appropriate framework to inform this present study ${ }^{16}$. The evaluation of the placement was consistent with Level I and II of Kirkpatrick's four levels of analysis ${ }^{11}$ : the participants' reaction to the placement and the degree to which they found the placement valuable (Level I: Reaction); and the extent to which participants acquired the intended knowledge and confidence (Level II: Learning).

Students were invited to provide informed consent prior to participation, and the study was approved by the Ethics Committee at Western Sydney University H13038 and was conducted during the first quarter of 2019.

\section{Results}

A total of 34 out of the 44 students who attended the placement completed the survey. The results of the survey are shown in Table 1. 
Table 1. Results of the student survey

\section{Statement}

$\%$ agree or

strongly agree

I feel more confident in my ability to assess a patient with a complex foot problem after this placement

I now have a better understanding of the scope of practice of a podiatrist

This placement was a useful learning experience

I had an overall sense of wellbeing whilst on placement

I felt valued as a learner during the placement

I would consider returning to the clinic as an employee
$88 \%$

$94 \%$

$91 \%$

$91 \%$

$91 \%$

$83 \%$
Analyses revealed that $88 \%$ of students believed they had a higher degree of confidence in their ability to assess patients with complex foot problems such as wounds following the observational placement. $94 \%$ of students identified that, following the placement, they gained a "better understanding of the scope of practice of a podiatrist". $91 \%$ of participants indicated that the observational placement was a useful learning experience and their free text answers indicated that the placement enabled them to consolidate learning and gain insight into a high risk foot clinic environment (Table 1). Student highlights of the observational placement were also supported by their free text answers in the post-placement survey (Tables $2 \mathrm{~A}$ and $\mathrm{B}$ ).

Further survey responses revealed that $91 \%$ of students agreed that they had an overall sense of wellbeing whilst being on the placement, and that this experience made them feel valued as learners. $83 \%$ declared that they would consider returning to work in a similar clinic environment as an employee, indicating an overall positive experience amongst the respondents.

\section{Discussion}

The approach evaluated in this study, where students observed clinical interactions without the burden of responsibility for patient care, was shown to be an effective way to increase student confidence in complex clinical situations. Previous work has emphasised the need to address student confidence and anxiety during clinical placement to mitigate adverse impacts on learning ${ }^{8}$. From a neuroscientific stand point, observational practice is believed to trigger the same neural connections that would be activated if the actions were being completed by the individual, known as the "mirror neuron system theory"17. Therefore, by shadowing clinicians in their work environments, students are able to consolidate learning in a quasi-applied way.

Early affective contextual learning whilst on placement has been shown to have positive long-term impacts on the students' ability to develop skills on subsequent placements by fostering a deeper understanding and reinforcement of theory ${ }^{1}$. Contextual learning in an engaging environment

Table 2A. Examples of 3rd year podiatry students' free text answers which illustrate the benefit of the observational placement in enabling "A greater understanding of podiatric scope of practice"

"gain a better understanding of how podiatry works in a hospital setting and how a high risk foot clinic operates and adds to the podiatric scope"

"seeing different aspects of podiatry"

"experiencing new scenarios which we otherwise wouldn't see in the student-run clinics"

Table 2B. Examples of 3rd year podiatry students' free text answers articulating how the observational placement contributed to "Consolidation of learning"

"being able to observe conditions discussed in lectures"

"being able to meet patients living with high risk feet and hearing their experiences - it really opened my eyes about how these patients are trying to live their lives and how living situations influence treatment plans"

"observing patients and observing the professional standard displayed by the health professionals"

"learning from the podiatrists without the pressure of treating patients"

"witnessing varied types of patients; how different each case is" 
allows students to apply knowledge gained didactically in a more effective way ${ }^{18}$. Students recognised that this placement increased their confidence levels in being able to assess complex foot conditions such as wounds, a skill that is difficult to teach didactically yet is paramount for proper acquisition of clinical skill and competence ${ }^{5}$.

The consolidation of learning on observational placements also provided students with tangible experiences to complement the theoretical learning undertaken throughout the unit; this was confirmed when students were asked about the highlights of their experiences as noted in Table 2B. The answers indicate that observational placement may add another dimension to student learning through experiencing a holistic approach to patient management that is difficult to learn didactically. Other answers indicated that observational placements may also assist in increasing exposure to the complex case and wound management undertaken in a clinical environment which are otherwise not effectively taught in a traditional classroom setting.

Traditionally, podiatry students are not placed at high risk foot clinics until the latter stages of their degree when they have had time to acquire the skill set necessary to treat complex presentations. In this study, $83 \%$ of students surveyed stated that they would like to return to work in the clinic as an employee, indicating a high level of interest within the specialty and there was an overwhelming consensus that students had a better understanding of the scope of their chosen profession. This is supported by evidence that suggests that early exposure to medical speciality areas through observation fosters interest in specific fields ${ }^{19}$.

With limited resources and time, public health departments are faced with the challenge of accommodating students whilst delivering quality patient care. The implementation of an observational placement was deemed less onerous than traditional hands-on student placement (personal communication with Head of Department) as learning does not require such close supervision and social distancing can be more easily maintained. There is also clear evidence from this survey that students gain confidence through observing in clinical environments, and therefore it is proposed that this more efficient model of placement has merit.

\section{Limitations of the study}

The authors acknowledge limitations of the study. The sample size $(n=34)$ was relatively small, reducing the overall power of the study. Survey results were obtained from only one clinical placement site and therefore may not be generalisable to all clinical placements. The placement was only half a day in total for each student - it could be contended that the participants had not had adequate time to be immersed in the clinical environment to comprehensively comment on outcomes. In addition, the effect of the placement in reducing student anxiety, which has been identified previously as a key stressor on clinical placement, was not measured. This would be considered for inclusion in future work together with a pre-placement survey to more comprehensively capture the benefits of this observational placement.

\section{Conclusion}

The data presented indicates observational placement is an appropriate method to introduce students to complex clinical environments, to enhance learning and to bridge the gap between theoretical knowledge and practical application whilst minimising disruption to clinical operations. The value of observation-only placements has been demonstrated in the discipline of podiatry; this could be useful in other disciplines as it is less resource intensive, improves student confidence through increasing familiarity to the clinical environment, and enables students to gain a better understanding of the scope of practice in their chosen profession.

\section{Ethics}

This work was undertaken with the permission of the Western Sydney University Human Ethics Committee \# H13038.

\section{Acknowledgements}

The authors would like to thank the staff at the high risk foot clinic at Royal North Shore Hospital, Sydney, where the placement took place. The enthusiasm of this highly dedicated team enabled the study to be undertaken.

\section{Conflict of interest}

The authors declare no conflicts of interest.

\section{Funding}

The authors received no funding for this study.

\section{References}

1. Currie G, Wheat J. The first year clinical placement for undergraduate medical radiation science students: tool or toil? Radiographer: The Official Journal of the Australian Institute of Radiography. 2005;52(2):18-22.

2. Roling G, Lutz G, Edelhäuser F, et al. Empathy, well-being and stressful experiences in the clinical learning environment. Patient Educ Couns 2020;103(11):2320-2327.

3. Beltrán-Velasco Al, Ruisoto-Palomera P, Bellido-Esteban A, García-Mateos M, Clemente-Suárez VJ. Analysis of psychophysiological stress response in higher education students undergoing clinical practice evaluation. J Med Syst 2019;43(3):68.

4. Baraz S, Memarian R, Vanaki Z. Learning challenges of nursing students in clinical environments: a qualitative study in Iran. J Educ Health Promot 2015;4:52-52.

5. Crooks D, Carpio B, Brown B, Black M, O'Mara L, Noesgaard C. Development of professional confidence by post diploma baccalaureate nursing students. Nurse Educ Pract 2005;5(6):360367.

6. Sharif $F$, Masoumi S. A qualitative study of nursing student experiences of clinical practice. BMC Nursing 2005;4(1):6.

7. Raymond JM, Medves JM, Godfrey CM. Baccalaureate nursing students' confidence on patient safety. J Nurs Ed Pract 2017;7(6). 
8. Panduragan SL, Abdullah N, Hassan H, Mat S. Level of confidence among nursing students in the clinical setting. Procedia - Soc Behav Sci 2011;18:404-407.

9. Australian and New Zealand Podiatry Accreditation Standard (ANZPAC). ANZPAC Podiatry Accreditation Standards (Version 5.1.2015).

10. Reio TG, Rocco TS, Smith DH, Chang E. A critique of Kirkpatrick's evaluation model. New Horizon Adult Ed Human Res Dev 2017;29(2):35-53.

11. Kirkpatrick D. Evaluation of training. In: Craig RL, editor. Training and development handbook: a guide to human resource development. New York: McGraw Hill; 1976.

12. Smidt A, Balandin S, Sigafoos J, Reed VA. The Kirkpatrick model: a useful tool for evaluating training outcomes. J Intellect Dev Disabil 2009;34(3):266-274.

13. Ahanchian M, Sharafi S, Vafaee M, Hajiabadi F. Evaluate the effectiveness of internship program in nursing student using Kirkpatrick's model. Res Med Ed 2017;9:17-19.

14. Maddineshat M, Hashemi M, Besharati R, Gholami S. The effectiveness of clinical teaching of mental health courses in nursing using clinical supervision and Kirkpatrick's model. Electronic Phys 2018;10:6265-6272.

15. Cooper S, Cant R, Waters D, et al. Measuring the quality of nursing clinical placements and the development of the Placement Evaluation Tool (PET) in a mixed methods co-design project. BMC Nursing. 2020;19(1):101.

16. Kirkpatrick D, Kirkpatrick J. Evaluating training programs: The four levels. Berrett-Koehler Publishers; 2006.

17. di Pellegrino G, Fadiga L, Fogassi L, Gallese V, Rizzolatti G. Understanding motor events: a neurophysiological study. Experiment Brain Res 1992;91(1):176-180.

18. Lave J, Wenger E. Situated learning: legitimate peripheral participation. Cambridge: Cambridge University Press; 1991.

19. Are C, Stoddard HA, Northam LC, Thompson JS, Todd GL. An experience in surgical anatomy to provide first-year medical students with an early exposure to general surgery: a pilot study. J Surg Educ 2009;66(4):186-189. 